\title{
Passenger Flow Analysis for the Surrounding Subway Stations of Large Special Event Site during Dissipation
}

\author{
Lu Zhao, a, En-jian Yao ${ }^{1, b}$,Sha-sha Liu ${ }^{1, \mathrm{c}}$ and Bin-bin Li, ${ }^{1, \mathrm{~d}}$ \\ ${ }^{1}$ MOE Key Laboratory for Urban Transportation Complex Systems Theory and Technology, Beijing \\ Jiaotong University, Beijing, 100044, China

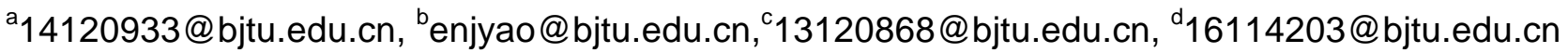

Keywords: urban rail transit; travel demand forecast; large special event; disaggregate model

Abstract. Urban rail transit plays an increasingly important role in conveying passengers when large special events are held. In order to analyze the impact of large special events on surrounding subway stations and provide the reasonable reference for the management department to organize passenger flow and make the train dispatching plan, it's necessary to analyze the passenger flow demand for the surrounding subway stations of large special event site during dissipation. First, the impact of large special events on entrance passenger flow of surrounding subway stations is analyzed. Then, using disaggregate theory, a model is established and estimated on the basis of stated preference survey data. Finally, based on the proposed model, the passenger flow of the surrounding stations during an actual event is forecasted. The result shows that the maximum relative error of total passenger flow is $8.4 \%$, verifying the proposed approach is to be practical.

\section{Introduction}

The accumulation of passenger flow caused by the large special events has brought huge passenger management pressure to the surrounding stations around the large special event site, especially when the sudden crowded passenger flow gathered and dissipated in a one-time special event. In the existing methods of passenger flow prediction for urban railway station's entrance and exit, many scholars use the ARIMA (Autoregressive Integrated Moving Average Model) model [1-3]. In addition, there are some methods such as trend analysis and regression analysis methods which use the region's past traffic or economic indicators etc. of the research areas [4-5]. However, the above methods rely on a large number of historical data, and a large number of passenger flow data under the same type of large special events are difficult to obtain.Therefore, this paper aims to build a passenger flow forecasting method considering the travel behavior of passengers during the large special event dissipation in the multi-station scenario.

\section{Impact Analysis of Large Special Events on Entrance Passenger Flow}

Construction of Direction Relevance Indicator. This paper introduces the direction relevance indicator among the directions of the origin station, the destination station, and the leaving space to analyze the impact of the passenger flow on the surrounding rail transit stations. When a passenger is away from the gate in the venue and $\alpha$ and $\beta$ satisfy equation(1), there is consistency among those three directions; otherwise, there is no consistency among those three directions.

$$
\left\{\begin{array}{l}
\alpha \in\left[0^{\circ}, 90^{\circ}\right] \\
\beta \in\left[0^{\circ}, 90^{\circ}\right]
\end{array}\right.
$$

Where, $\alpha$ is the angle between the leaving direction and the origin station direction, and $\beta$ is the angle between the origin station direction and the destination station direction

Impact of Entrance Passenger Flow. Based on the passenger flow features on activity days and ordinary days of the stations surrounding the place where large special events are being held, this paper obtains the data of regular entrance passenger flow per 15 minutes on activity days of DONGSISHITIAO and TUANJIEHU as shown in Fig.1. It can be seen from Fig.1 that the passenger 
flow of the stations surrounding the place when the large special event is being held is massively larger than that on ordinary days, and it has the large passenger flow features with concentrated gathering time.
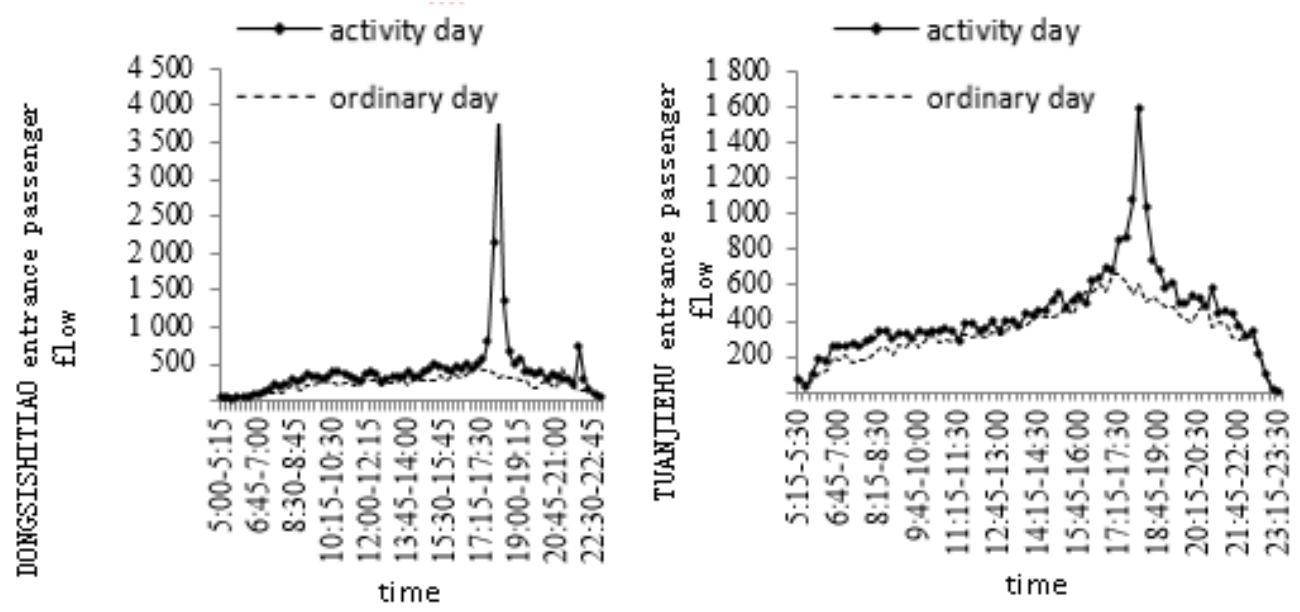

Fig.1 Entrance passenger flow of surrounding rail transit stations

\section{Entrance Passenger Flow Forecasting Model during Dissipation}

The station choice is influenced by the factors including directional relevance, walking time, utilization time of rail transit, degree of station congestion and other factors adopted as the characteristic variables of utility functions, with all the factors the MNL (Multi-nominal Logit) model established. When the random error item $\varepsilon_{i j k n}$ conforms to the independent Gumbel distribution[6], for an individual $n$, departing from the letting-out door $i$ to the destination station at the direction of $k$, the probability of choosing the origin station $j$ and the utility function are as shown in equation (2) and (3).

$$
\begin{gathered}
P_{i j k n}=\frac{\exp \left(V_{i j k n}\right)}{\sum_{j \in B_{n}} \exp \left(V_{i j k n}\right)},\left(i \in A_{n}, j \in B_{n}, k \in C_{n}\right) \\
V_{i j k n}=\theta_{1} F X_{i j k n}+\theta_{2} T_{i j n}^{\text {walk }}+\theta_{3} T_{j k n}^{\text {take }}+\theta_{4} C_{j n}
\end{gathered}
$$

In equation (2), $P_{i j k n}$ is the probability of traveler $n$ departing from the letting-out door $i$ to the destination station at the direction of $k$ and choosing station $j$ to get in; $V_{i j k n}$ is a fixed item of the utility function; $A_{n}$ is the choice set of letting-out doors of traveler $n ; B_{n}$ is the origin station choice set of traveler $n ; C_{n}$ is the destination station direction set of traveler $n$. In equation $(3), \theta_{l}(i=1,2,3,4)$ is an undetermined coefficient; $F X_{i j k n}$ is the variate (0-1) of the directional relevance of traveler $n$ walking form the letting-out door $i$ to origin station $j$ to take the rail transit to the destination station at the direction of $k$, and when the directions are the same, it is 1 ; otherwise it is $0 . T_{i j n}^{\text {walk }}$ is the walking time of traveler $n$ from the letting-out door $i$ to origin station $j . T_{j k n}^{\text {take }}$ is the rail transit utilizing time of traveler $n$ from the origin station $j$ to the destination station $k . C_{j n}$ is the variate $(0-1)$ of the degree of congestion of station $j$, and when it is congested, it is 1 ; otherwise it is 0 .

The passenger flow distribution proportion of each of the surrounding rail transit station are as shown in equation (4) and (5).

$$
\begin{aligned}
O_{j} & =\sum_{i=1}^{A_{n}} \sum_{k=1}^{C_{n}} O_{i j k}=\sum_{i=1}^{A_{n}} \sum_{k=1}^{C_{n}} \sum_{n=1}^{q_{i k}} P_{i j k n}=\sum_{i=1}^{A_{n}} \sum_{k=1}^{C_{n}} q_{i k} \cdot P_{i j k} \\
& =\sum_{i=1}^{A_{n}} \sum_{k=1}^{C_{n}} Q \cdot P_{\text {rail }} \cdot P_{Q_{i}} \cdot P_{Q_{k}} \cdot P_{i j k}
\end{aligned}
$$




$$
P_{O_{j}}=\frac{O_{j}}{\sum_{j \in B_{n}} O_{j}}
$$

In equation (4) and (5), ${ }^{O_{j}}$ is the total population of passenger flow arriving at station $j$ when dissipation; ${ }^{P_{O_{j}}}$ is the proportion of the passengers choosing the origin station $j$ to the total passengers of all the stations; ${ }^{O}{ }_{i j k}$ is the population departing form the letting-out door $i$ to get in to station from $j$ to $k ;{ }^{P_{i j k}}$ is the result of the repeated experimental calculation of $P_{i j k n}$, and it can be known form the theory of probability statistics that $P_{i j k}=P_{i j k n} . q_{i k}$ is the passenger flow departing form the letting-out door $i$ to the destination station at the direction of $k ; Q$ is activity participants; $P_{\text {rail }}$ is the utilization ratio of rail transit; $P_{Q_{i}}$ is the passenger flow ratio departing from the letting-out door $i$; $P_{Q_{k}}$ is the passenger flow ratio of destination station $k$.

With SP (Stated Preferanse) questionaire and Maximum Likelihood estimations on the undetermined parameters of utility functions, the results are as shown in Table 1.

Tab.1 Model estimation results

\begin{tabular}{ccc}
\hline Parameters & Estimates & Est./s.e. \\
\hline direction relevance & 0.8189 & 10.711 \\
walking time/10min & -2.0199 & -16.499 \\
utilization time of rail transit/10min & -0.8740 & -8.148 \\
degree of station congestion & -0.3070 & -14.517 \\
\hline$\rho^{2}$ & & 0.2111 \\
sample size & & 1860 \\
\hline
\end{tabular}

The results above mean the modal has good accuracy, and describes passengers' station choice behaviors well.

\section{Case Analysis}

By using the model and combining with the background of Super League Sport Event which happened in the Beijing Workers' stadium on November 3, 2013, this paper selects the surrounding rail transit stations as the research object to forecast. The result is shown in Figure 2.

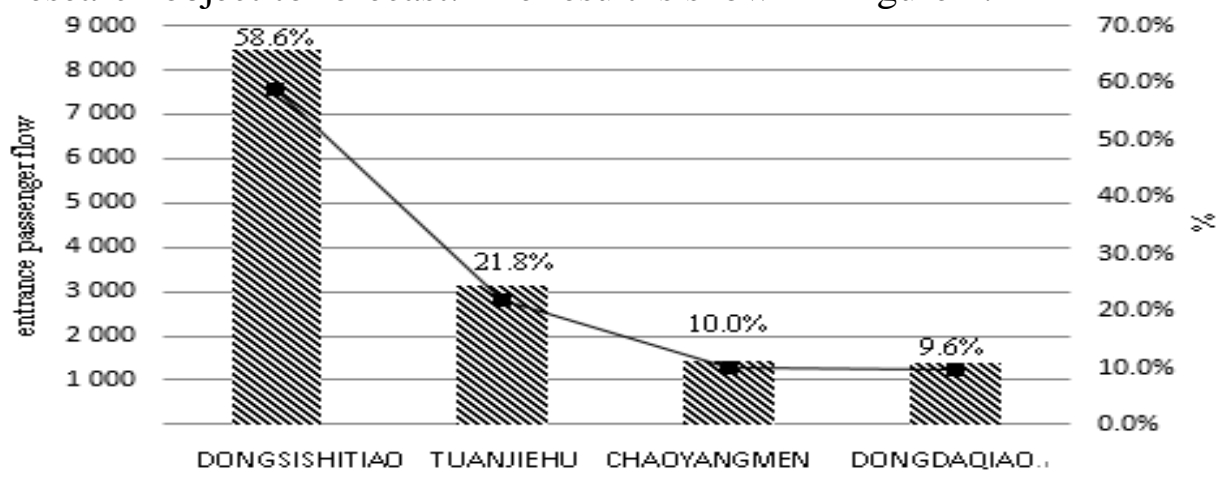

Fig. 2 Forecast of entrance passenger flow distribution

It can be seen from Figure 2 that on the day of the event, DONGSISHITIAO is predicted to have a large number of passengers who participate in the large special event.

Prediction Accuracy Verification of the Model. According to the predicted space distribution of entrance passenger flow, the real historical data is combined to conduct error analysis and inspection of the predicted results. This paper uses the ARIMA (Auto Regressive Integrated Moving Average) model to analyze the time series data related to the entrance passenger flow, and compares it with the forecasting model which is established in this paper. By obtaining the difference value between the 
regular entrance passenger flow of the event day which is sought and predicted via ARIMA (Auto Regressive Integrated Moving Average) model and the time-shared card-swiping entrance passenger flow data of each station in Beijing rail transit center, space distribution results of the entrance passenger flow who participate in the event at each station can be obtained. This paper compares the real entrance passenger flow who participate in the event and the predicted value of MNL model to calculate the prediction error. The error results of the model are shown in Table 2.

Tab.2 Error results of the prediction based on the model

\begin{tabular}{ccc}
\hline & Absolute Error & Relative Error \\
\hline DONGSISHITIAO & 79 & $0.9 \%$ \\
TUANJIEHU & 33 & $1.1 \%$ \\
CHAOYANGMEN & 61 & $4.1 \%$ \\
DONGDAQIAO & 107 & $8.4 \%$ \\
\hline
\end{tabular}

It can be seen from Table 2 that the maximum relative error between the predicted value of passenger flow forecasting model constructed in this paper and the real value is $8.4 \%$. Thus, it can be determined that the prediction has a high accuracy and the prediction results have good applicability.

\section{Conclusions}

The urban rail transit passenger flow prediction model under the multi-station situation which is constructed in this paper can provide some references for development of the passenger flow evacuation and the passengers induction schemes for the surrounding urban rail stations during the large special event.

\section{Acknowledgements}

This work was financially supported by National 973 Program of China (No. 2012CB725403-1)

\section{References}

[1] Changjun Cai, Enjian Yao, Meiying Wang, et al. Prediction of urban railway station's entrance and exit passenger flow based on multiply ARIMA model[J]. Beijing: Journal of Beijing Jiaotong University,2014, 38(02):135-140. (in Chinese)

[2] Bingfeng Si, Jiuran He, Hualing Ren, et al, Urban railway traffic passenger flow forecast based on the timing characteristics [J]. Beijing: Journal of Beijing Jiaotong University, 2014,38(3):1-6.

[3] Chao Han, Su Song, Chenghong Wang, et al. A real-time shot-term traffic flow adaptive forecasting method based on ARIMA mode [J]. Journal of System Simulation, 2004,16(7):1530-1532,1535. (in Chinese)

[4] Jun Yang, Zhongsheng Hou. A wavelet analysis based LS-SVM rail transit passenger flow prediction method [J]. China Railway Science. 2013,34(3):122-127. (in Chinese)

[5] Chunfu Shao. Traffic planning [M]. China Railway Publishing House, 2004.4. (in Chinese)

[6] Hongzhi Guan. Disaggregate model: tool of traffic behavior analysis[M].China Communications Press,2004:10-11. (in Chinese) 